\title{
Development of Analytical Method for In Vitro Release Testing of Dexamethasone Nanosuspensions
}

\author{
Antonia Šutić ${ }^{1}$, Marieta Duvnjak Romić2 ${ }^{2}$ Sandra Miočić², and Biserka Cetina-Čižmek ${ }^{2 *}$ \\ ${ }^{1}$ Faculty of Pharmacy and Biochemistry University of Zagreb, Zagreb, Croatia \\ ${ }^{2}$ PLIVA Croatia Ltd., Research and Development, Zagreb, Croatia
}

e-mail: biserka.cetina-cizmek@pliva.com

\begin{abstract}
The aim of this study was to develop and compare analytical methods for in vitro release testing (IVRT) of an active substance, dexamethasone, from nanosuspension using three apparatuses for IVRT of topical formulations (immersion cell, vertical diffusion cell, and dialysis bag membrane methods), taking into account the physiological conditions at the point of application of these therapeutic systems. Sensitive and reproducible methods to assess in vitro release rates were developed, and immersion cell method was chosen as most appropriate for evaluation of dexamethasone release.
\end{abstract}

KEYWORDS: Dexamethasone, nanosuspension, in vitro release testing (IVRT), immersion cell, vertical diffusion cell, dialysis bag membrane method

\section{INTRODUCTION}

T here has been an increasing focus on evaluation and research of new routes for dexamethasone administration to provide targeting of the drug for local action, and consequently reduce systemic side effects and avoid the first pass metabolism through the liver. For topical administration of dexamethasone, it is necessary to develop an appropriate therapeutic system that would enable sustained and controlled release of the drug.

Topical drug delivery systems serve as carriers for drugs that are delivered by skin and mucosa. Because of their peculiar rheological behavior, topical formulations can adhere to the tissue surface for sufficiently long periods, which helps prolong drug delivery at the application site. A topical drug delivery system is advantageous in terms of its easy application, simple formulation, and ability to deliver a wide variety of drug molecules (1).

In the last decades, a great attention has been paid to the development of nanoscale systems, new drug delivery systems, based on nanoparticles that would overcome the deficiencies of existing conventional delivery systems. Some of the most promising drug delivery nanoscale systems are pharmaceutical nanosuspensions. A pharmaceutical nanosuspension is defined as very finely colloid, biphasic, dispersed solid drug particles in an aqueous vehicle, size below $1 \mu \mathrm{m}$, stabilized by surfactants and polymers, and prepared by suitable methods for drug delivery applications. Nanosuspensions provide efficient delivery of hydrophobic drugs and increases the bioavailability (2).

High-pressure homogenization is the relatively simple, most widely used method for the preparation of nanosuspensions of many poorly water-soluble drugs (3). Microfluidizer technology, a type of high-pressure homogenization that relies on pumping a drug through micron-sized orifices under the high pressure, was used to prepare dexamethasone nanosuspensions in the present study.

The purpose of a performance test for a dosage form is to predict and monitor the consistency in manufacturing and quality of that dosage form. In vitro release testing (IVRT) represents a useful tool during the development of a topical dosage form. Monitoring the release of a drug from its dosage form during clinical trials can be critical in understanding the efficacy of the formulation and can be successful in establishing an in vitro-in vivo correlation for topical products (4).

IVRT methods should be reproducible and sensitive to small changes in physicochemical properties of the

*Corresponding author 
dosage form (5). Development of IVRT tests for the nanosuspension delivery systems is especially challenging due to difficulties with sample filtration and/or separation.

In the present study, IVRT methods have been developed for evaluation of in vitro release of dexamethasone from a nanoparticle-based therapeutic system using three different apparatuses for testing of topical formulations (immersion cell, vertical diffusion cell, and dialysis bag membrane method). Discriminatory power of the methods related to differences in drug loading and particle size was tested, as well as repeatability of the measurements. Pros and cons for each method were recognized, and the most appropriate IVRT method for evaluation of dexamethasone release was identified.

\section{MATERIALS AND METHODS \\ Materials}

Citric acid, disodium phosphate, ethanol, and potassium dihydrogen phosphate were purchased from Kemika d.d., Croatia. Micronized dexamethasone (particle size, d90 = $10 \mu \mathrm{m}$ ) was obtained from Pfizer, Inc., USA. MONTANOX Polysorbate 20 was purchased from SEPPIC S.A., France, tyloxapol from Albany Molecular Research, Inc., USA, and Blanose 7MF sodium carboxymethyl cellulose (CMC) from Ashland Global Holdings, Inc., USA. Sodium chloride, hydrochloric acid, sodium hydroxide, and acetonitrile were obtained from Merck, Germany.

\section{Preparation of Nanosuspension}

Micronized dexamethasone was homogeneously suspended in an aqueous solution of a surfactant, tyloxapol, using an T 25 ULTRA-TURRAX disperser (IKA-Werke GmbH \& Co. KG, Germany). The obtained concentrate was homogenized using a Biopharmaceutical Microfluidizer Processor M-110EH-30 (Microfluidics Corp., USA) at pressure of $1.8 \times 10^{5} \mathrm{kPa}$. As a part of formulation and process optimization, the number of homogenization cycles $(30,40$, and 50$)$ was varied, as was the concentration of dexamethasone $(1 \%, 5 \%)$ and tyloxapol-surfactant $(0.5 \%, 2.5 \%)$. After homogenization, dexamethasone concentrates were diluted with a $\mathrm{CMC} /$ sodium chloride aqueous solution to obtain the final formulation ( $0.1 \%$ dexamethasone, $0.5 \% \mathrm{CMC}$, and $0.9 \%$ sodium chloride). Hydrochloric acid and sodium hydroxide aqueous solutions were used for $\mathrm{pH}$ adjustment to 7.4. Particle size distribution of formulations was analyzed by low-angle laser light scattering (LALLS). Based on the obtained particle size results, 40 homogenization cycles of $1 \%$ dexamethasone concentrate containing $0.5 \%$ tyloxapol were selected for the preparation of the nanosuspension.

\section{Equilibrium Solubility of Dexamethasone in Mcllvaine Buffer}

The equilibrium solubility of dexamethasone was determined by the shake flask method. As thermodynamic solubility was measured, particle size of tested substance is not relevant parameter. Therefore, micronized dexamethasone was tested in this experiment $(\mathrm{d} 90=$ $10 \mu \mathrm{m}$ ). Solubility in Mcllvaine buffer (buffer solution composed of citric acid and disodium hydrogen phosphate) with addition of different amounts of ethanol (EtOH) $(0 \%, 10 \%$, and $20 \%$; v/v) was determined. The analysis was carried out in triplicate for each combination of active pharmaceutical ingredient (API) and medium. Dexamethasone was added in surplus to medium and shaken under predetermined conditions: $24 \mathrm{~h}, 75 \mathrm{rpm}$, and $32{ }^{\circ} \mathrm{C}$. The saturation was confirmed by observation of the presence of undissolved material. A $0.45-\mu \mathrm{m}$ filter was used for separation. After filtration, the samples were taken for analysis and diluted to prevent recrystallization. The amount of solute contained in the samples was determined by high-performance liquid chromatography (HPLC).

\section{HPLC Analysis}

Quantification of dexamethasone in the samples was determined by HPLC (Agilent 1100/1200 Infinity Diode Array Detector, Agilent Technologies, Inc., USA). Separation was performed on a Kinetex C18 $4.6 \times 50 \mathrm{~mm}$ column packed with $2.6-\mu \mathrm{m}$ particles and a pore size of $100 \AA$ (Phenomenex, Inc., USA), maintained at $25^{\circ} \mathrm{C}$. The injector temperature was set at $20^{\circ} \mathrm{C}$. Injection volume was $100 \mu \mathrm{L}$ and chromatograms were recorded at $241 \mathrm{~nm}$. Mobile phase consisted of 70\% phosphate buffer $\mathrm{pH} 2.0$ and $30 \%$ acetonitrile with an isocratic flow rate of $1.5 \mathrm{~mL} /$ $\min$.

\section{Evaluation of the Suitability of the Membranes}

In the present study, three membranes were tested: a 0.1- $\mu \mathrm{m}$ polyethersulfone (PES) membrane (Pall Corp., USA) and 50- and 300-kDa cellulose ester (CE) dialysis membranes. The membranes were soaked in a solution of the API in the receptor medium: Mcllvaine buffer with $10 \%$ EtOH and Mcllvaine buffer with $0.3 \%$ tyloxapol (surfactant present in the formulation). Membrane-binding studies were performed to determine whether the API binds to a specific membrane. API binding to membrane (recovery) was determined by HPLC analysis in comparison to API solutions in which membranes were not soaked. 


\section{In Vitro Testing \\ Immersion Cells}

In vitro dissolution was carried out via USP apparatus 2 (paddle) with the small spin-paddles at a speed of $100 \mathrm{rpm}$ in $75 \mathrm{~mL}$ of receptor medium (Mcllvaine buffer $\mathrm{pH}$ 7.4/ ethanol; $90 / 10(\mathrm{v} / \mathrm{v}))$ maintained at $32{ }^{\circ} \mathrm{C}$. Analysis of each sample was performed in sextuplicate. PES membranes (previously soaked in the receptor medium for at least 30 $\mathrm{min}$ ) with a pore size of $0.1 \mu \mathrm{m}$ were selected as the barrier. Dexamethasone nanosuspension samples in volumes of 1 $\mathrm{mL}$ were applied in the donor chamber of the immersion cell. Membranes were carefully placed over the top of the sample compartment to minimize the possibility of air bubble formation between the surface of the sample and the membrane. After the immersion cell components were assembled, the completed assembly was carefully placed into the bottom of the dissolution vessel with the membrane facing up. Sampling was performed manually at $20,40,60,80,100,120,140,160$, and $180 \mathrm{~min}$, in a volume of $1 \mathrm{~mL}$ without replacement with fresh medium. Drug concentrations were quantified using HPLC.

\section{Dialysis Bag Method}

A magnetic stirrer (IKA-Werke GmbH \& Co. KG, Germany) with the ability to regulate temperature was used to control rotation and heating of the system. Analysis of each sample was performed in sextuplicate. Mcllvaine buffer $\mathrm{pH} 7.4$ was used as a receptor medium in a volume of $200 \mathrm{~mL}$ per each glass and it was maintained at $32{ }^{\circ} \mathrm{C}$.

CE dialysis membranes (300-kDa) were previously soaked in the receptor medium for at least $30 \mathrm{~min}$. A volume of $1 \mathrm{~mL}$ of dexamethasone nanosuspension was put in a dialysis bag (total surface area of $960 \mathrm{~cm}^{2}$ ). Both ends were tied. Dialysis bags with samples were carefully placed in beakers filled with thermostated medium. The speed of the magnetic stirrer was $400 \mathrm{rpm}$. Sampling was performed manually at 5, 10, 15, 20, 30, 40, 50, 60, 75, and $90 \mathrm{~min}$, in a volume of $1 \mathrm{~mL}$ without replacement of fresh medium. Drug concentrations were quantified using HPLC.

\section{Vertical Diffusion Cells}

An automatic Franz cell system (Vision Microette, Teledyne Hanson Research, Inc., USA) with six vertical diffusion cells of $12 \mathrm{~mL}$ (with an effective diffusion area of $1.7 \mathrm{~cm}^{2}$ ) was built using a PES membrane (previously soaked in the receptor medium for at least $30 \mathrm{~min}$ ) with a pore size of $0.1 \mu \mathrm{m}$, which was placed on the upper donor chamber of the diffusion cell, separating this compartment from the receptor chamber. Dexamethasone nanosuspension samples in volumes of $1 \mathrm{~mL}$ were applied in the donor chamber of the cell. The receptor medium was $80 / 20(\mathrm{v} / \mathrm{v})$ Mcllvaine buffer $\mathrm{pH}$ 7.4/ethanol. The temperature of the diffusion cells was maintained at $32{ }^{\circ} \mathrm{C}$. Analysis of each sample was performed in sextuplicate. The magnetic stirrer speed was $400 \mathrm{rpm}$. The tubes were rinsed with $1.5 \mathrm{~mL}$ of fresh receptor medium prior to sampling. Every hour for 13 hours a 1-mL sample was withdrawn automatically and replaced with fresh, prewarmed medium. Drug concentrations were determined using HPLC.

\section{Calculation of Rate and Amount of Drug Released}

Denoted as $A R_{n}$, the amount released $\left(\mu \mathrm{g} / \mathrm{cm}^{2}\right)$ at a given time $\left(t_{1}, t_{2}\right.$, etc.) is calculated for each sample as follows:

Amount released at

$$
t_{1} A R_{1}=\left(\frac{A_{U_{1}}}{A_{S}}\right) \times C_{S} \times 1000 \times\left(\frac{V_{C}}{A_{0}}\right) \quad \text { Eq. (1) }
$$

Amount released at

$$
\begin{aligned}
t_{2} A R_{2}= & \left(\frac{A_{U 2}}{A_{S}}\right) \times C_{S} \times 1000 \times\left(\frac{V_{C}}{A_{0}}\right)+\left[A R_{1} \times\left(\frac{V_{S}}{V_{C}}\right)\right] \quad \text { Eq. (2) } \\
A R_{\mathrm{n}}= & \left(\frac{A_{U n}}{A_{S}}\right) \times C_{S} \times 1000 \times\left(\frac{V_{C}}{A_{O}}\right)+\left[\left(\frac{V_{S}}{V_{C}}\right) \times \sum_{i=1}^{n-1}\left(\frac{A_{U(n-1)}}{A_{S}}\right)\right. \\
& \left.\times \operatorname{Cs} \times 1000 \times \frac{V_{C}}{A_{0}}\right]
\end{aligned}
$$

where $A R=$ amount of drug released per unit area of the membrane $\left(\mu \mathrm{g} / \mathrm{cm}^{2}\right)$;

$A_{U}=$ response (e.g., peak area, or peak height or absorbance) from the sample solution;

$A_{S}=$ average response (e.g., peak area, or peak height or absorbance) from the standard solution; $C_{S}=$ concentration of the standard solution $(\mathrm{mg} / \mathrm{mL})$;

$V_{C}=$ volume of the receptor medium $(\mathrm{mL}) ;$

$A_{O}=$ area of the membrane $\left(\mathrm{cm}^{2}\right) ;$ and

$V_{S}=$ sample volume $(\mathrm{mL})$.

For each cell, the individual amount of drug released per unit area of the membrane $\left(\mu \mathrm{g} / \mathrm{cm}^{2}\right)$ is plotted versus the square root of time $(t)$ according to the Higuchi model for drug release.

The cumulative amount of dexamethasone released was calculated and plotted against the square root of time. The slope of the resulting line represents the rate of drug release.

Each sample was analyzed six times. The average value for each sampling time and the standard deviation (SD) 
was calculated as a measure of dispersion of the results around the average value and relative standard deviation (RSD) was determined as the ratio of the standard deviation and average value (6).

To determine the repeatability of all selected methods, the average values of slopes (estimated in vitro release rates) for targeted $100 \%$ nanosuspensions, which were obtained by sensitivity testing on changes in concentration and particle size of the API, were used. The percentage difference was calculated for the average values of slopes (estimated in vitro release rates) according to the following formula:

$$
\frac{A B S \text { (Value 1-Value 2) }}{\text { AVG (Value 1,Value 2) }} \times 100
$$

where $A B S=$ absolute value; $A V G=$ average value; Value 1 = average value of slopes for targeted $100 \%$ nanosuspension, which were obtained by sensitivity testing on changes in concentration of the API; and Value 2 = average value of slopes for targeted $100 \%$ nanosuspension, which were obtained by sensitivity testing on changes of API particle size.

\section{RESULTS AND DISCUSSION}

\section{Preparation of Nanosuspension}

Nanosuspensions were effectively produced by high pressure homogenization. After 40 homogenization cycles, particles sizes for milled sample (d10 $=0.36 \mu \mathrm{m}$; $\mathrm{d} 50=0.61 \mu \mathrm{m} ; \mathrm{d} 90=1.03 \mu \mathrm{m}$ ) were approximately 6 times smaller compared to non-milled sample ( $\mathrm{d} 10=0.79 \mu \mathrm{m}$; $\mathrm{d} 50=2.66 \mu \mathrm{m} ; \mathrm{d} 90=6.65 \mu \mathrm{m}$ ). To evaluate discriminatory power of the methods for the differences in particle size, untreated and homogenized sample suspensions were used.

\section{Equilibrium Solubility and Determination of Release Medium}

The equilibrium solubility of dexamethasone was determined in the following medium: Mcllvaine buffer with different percentage compositions of ethanol $(0 \%, 10 \%$, and $20 \%, v / v)$. The equilibrium solubility of dexamethasone in Mcllvaine buffer was $0.084 \mathrm{mg} / \mathrm{mL}$, in Mcllvaine buffer with $10 \%$ ethanol it was $0.167 \mathrm{mg} / \mathrm{mL}$, and in Mcllvaine buffer with $20 \%$ ethanol it was 0.324 $\mathrm{mg} / \mathrm{mL}$.

Data shown in Table 1 indicate a comparison of concentrations in case of completely released API and theoretical concentrations of solute (API) required to achieve sink conditions for the selected methods.

With regards to the results of the equilibrium solubility testing and sink conditions, Mcllvaine buffer with 20\% ethanol was used as receptor medium for IVRT with vertical diffusion cell, Mcllvaine buffer with $10 \%$ ethanol was used as receptor medium for IVRT with immersion cell, and pure Mcllvaine buffer was selected as receptor medium for the dialysis bag method.

Table 1. Comparison of Concentrations of Solute (API) Required to Achieve Sink Conditions for the Selected IVRT Methods

\begin{tabular}{|c|c|c|}
\hline IVRT Method & $\begin{array}{c}\text { Maximum } \\
\text { Concentration }(\mathrm{mg} / \\
\mathrm{mL})\end{array}$ & $\begin{array}{c}\text { Theoretical } \\
\text { Concentrations of API } \\
(\mathrm{mg} / \mathrm{mL})\end{array}$ \\
\hline $\begin{array}{c}\text { Immersion Cell } \\
\text { Method }\end{array}$ & 0.013 & 0.040 \\
\hline Dialysis Bag Method & 0.005 & 0.015 \\
\hline $\begin{array}{c}\text { Vertical Diffusion Cell } \\
\text { Method }\end{array}$ & 0.083 & 0.250 \\
\hline
\end{tabular}

$A P I$, active pharmaceutical ingredient; IVRT, in vitro release testing.

\section{Suitability of the Membranes}

The membrane keeps the product and the receptor medium separate and distinct and represents the critical factor in development of the IVRT methods for nanosuspension delivery systems. Membranes are chosen to offer the least possible diffusional resistance and not to be rate controlling.

Membrane-binding studies were performed to determine whether the API binds to a specific membrane. Buffer with $10 \%$ of EtOH (middle alcohol amount) served as a representative of all three media used in the IVRT experiments. Recovery for the CE 300-kDa membrane was $98.6 \%$ in the $10 \%$ EtOH medium and $98.2 \%$ in the $0.3 \%$ tyloxapol medium. For the CE $50-\mathrm{kDa}$ membrane, recovery was $101.8 \%$ and $99.7 \%$ in $10 \%$ EtOH and $0.3 \%$ tyloxapol, respectively. The result of API binding (recovery) to the PES $0.1-\mu \mathrm{m}$ membrane was $98.7 \%$ and $100.5 \%$ in $10 \% \mathrm{EtOH}$ and $0.3 \%$ tyloxapol. Based on these results, all the membranes tested were within the set requirements (recovery: 98.0-102.0\%) and were considered suitable for use in IVRT of dexamethasone.

PES 0.1- $\mu \mathrm{m}$ and CE 300-kDa membranes, with pore sizes smaller than the milled dexamethasone particle size in tested samples, were selected for further use. The CE 50-kDa membrane was excluded from further use due to diffusional resistance as a consequence of small pore size.

\section{Discriminatory Power of the Methods for Differences in Dosage Strength}

The IVRT method must be discriminatory, i.e., it should detect differences in release rates which may occur due to formulation changes or manufacturing process that could influence the product efficacy in vivo. In accordance 
with predetermined conditions, IVRT with immersion cell, vertical diffusion cell, and dialysis bag method was performed for three formulations with different dosage strengths of the dexamethasone nanosuspensions: $50 \%$, $100 \%$, and $150 \%$ API nanosuspensions. Data on the amount of drug released at predefined time intervals were obtained.

Figures 1, 2, and 3 depict the in vitro release profiles of the three formulations with different dosage strengths of the API nanosuspension (50\%, 100\%, and $150 \%$ ) that were obtained using immersion cells, vertical diffusion cells, and the dialysis bag membrane method. The dexamethasone release rate from the nanosuspension is represented by the slope of the line obtained from plotting amount of dexamethasone released per unit area of the membrane $\left(\mu \mathrm{g} / \mathrm{cm}^{2}\right)$ versus the square root of time ( $t$ ) (5). This release rate measure is formulationspecific and can be used to monitor product quality.

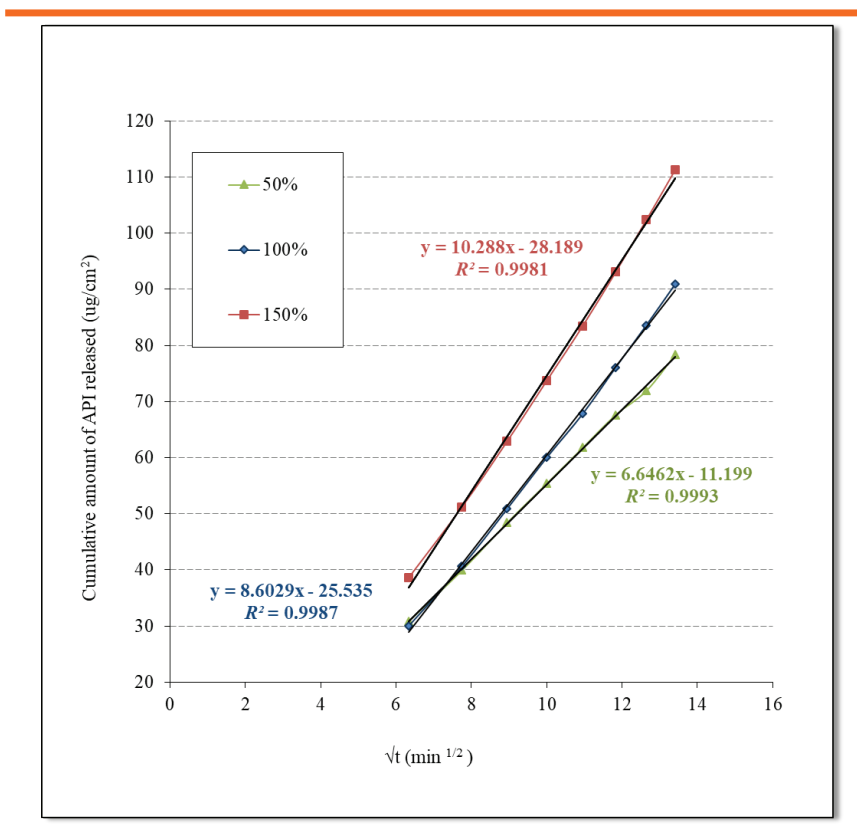

Figure 1. Drug release profiles from formulations at three dosage strengths that were obtained using immersion cells $(n=6)$. Black lines represent the slope. API, active pharmaceutical ingredient.

The slope in Figure 1 shows the difference in the release rate due to different dosage strength. The release rate of dexamethasone increases with increasing drug concentration in the formulation. This is expected because in the formulation with higher concentration of dexamethasone a greater amount of dexamethasone, which can diffuse through the membrane into the receptor medium, is available.

Six individual slopes (estimated in vitro release rates) obtained by testing a $100 \%$ API sample were compared with six individual release slopes (estimated in vitro release rates) of the $50 \%$ API sample, and then with six individual slopes (estimated in vitro release rates) of the $150 \%$ API sample.

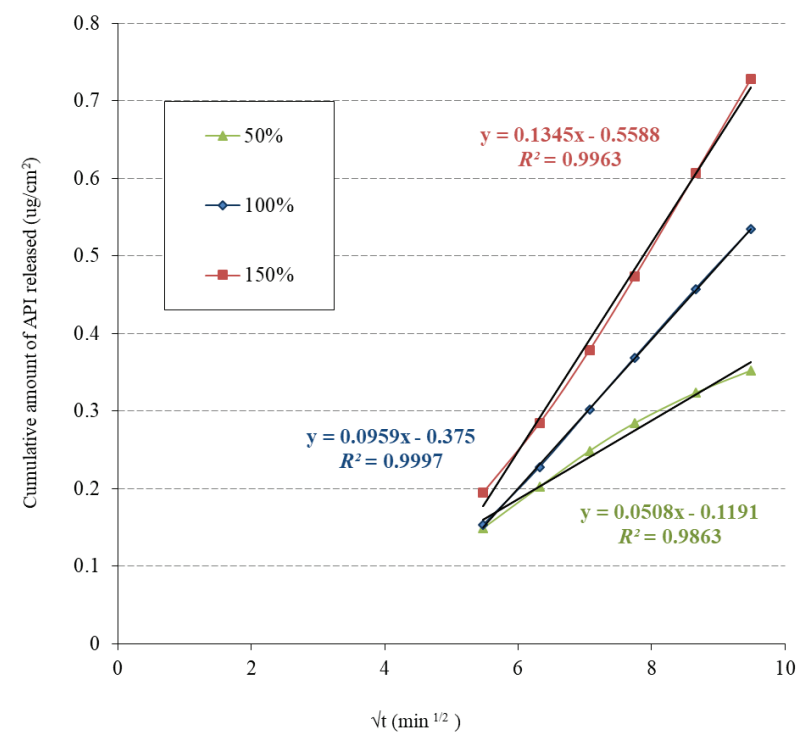

Figure 2. Drug release profiles from formulations at three dosage strengths that were obtained using dialysis bag method $(n=6)$. Black lines represent the slope. API, active pharmaceutical ingredient.

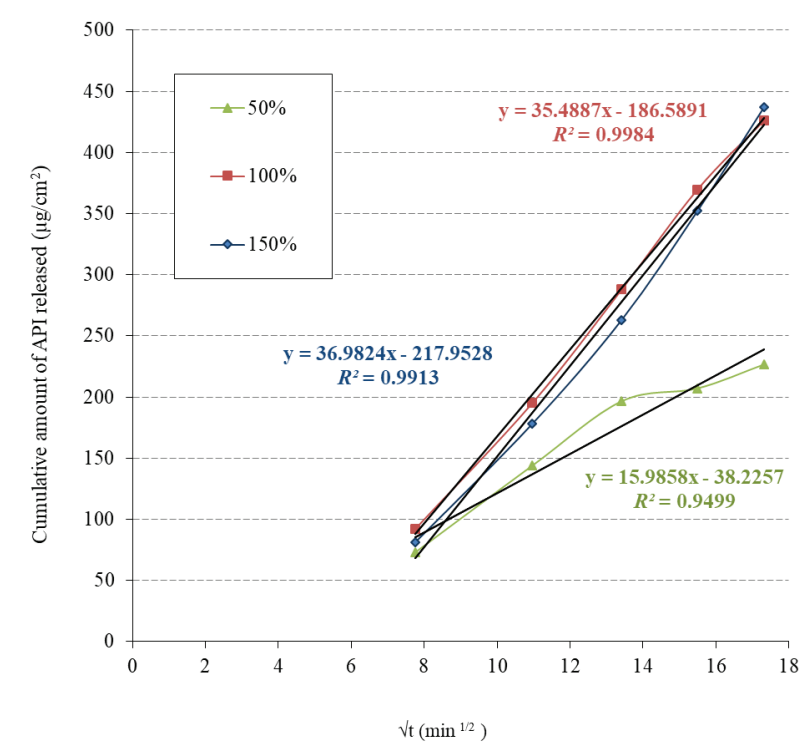

Figure 3. Drug release profiles from formulations at three dosage strengths that were obtained using vertical diffusion cells $(n=6)$. Black lines represent the slope. API, active pharmaceutical ingredient. 
According to the Scale-Up and Post-Approval Change Semisolid (SUPAC-SS) guideline, a 90\% confidence interval (Cl) of IVRT for two tested formulations should fall within the limits of $75 \%$ to $133.33 \%$ to declare two tested samples as similar, i.e., to have the same in vitro release rate of the API. If the $90 \% \mathrm{Cl}$ falls within the limits of $75 \%$ to $133.33 \%$, no further in vitro testing is necessary (7). Ninety percent $\mathrm{Cl}$ levels of IVRT with immersion cell, vertical diffusion cell, and dialysis bag method for three formulations with different dosage strengths of the API in nanosuspensions are shown in Table 2. Method with vertical diffusion cell was shown to be sufficiently sensitive to detect changes in concentration of the API only in the case of lower concentration ( $50 \%$ vs $100 \%$ ). When samples with high concentration were tested, no differences in release rates were observed. This behavior can be explained by the small surface of the membrane across which the diffusion of the therapeutic agent occurs. In case of product with higher drug concentration, membrane surface area represents limitation factor for drug release. The dialysis bag membrane method revealed sufficiently sensitive to the tested parameter, but great variability in the results was observed due to more consecutive steps in method performance and nonstandard set up of the method (e.g., unstandardized precut dialysis bag lengths and closures). Immersion cell method was shown as discriminative for changes in drug concentration, with low variability within the results.

\section{Release Rate Dependence on Particle Size}

In accordance with predetermined conditions, IVRT was performed using immersion cell, vertical diffusion cell, and dialysis bag methods for two formulations with different API particle size - milled (d90 $1.0 \mu \mathrm{m})$ and non-milled (d90 $6.65 \mu \mathrm{m}$ ) samples. Data on the amount of the drug released at predefined time intervals were obtained (Fig. 4).

Six individual slopes (estimated in vitro release rates) obtained by testing formulation with larger particle size was compared with six individual slopes (estimated in vitro release rates) of the formulation with smaller particle size. A $90 \% \mathrm{Cl}$ for comparison of these formulations was $70.25 \%-78.87 \%$ for immersion cell method and 73.60\%-112.35\% for dialysis bag method. Therefore, it can be concluded that these methods are sensitive to changes in API particle size. The first step of similarity requirement between two tested formulations was met in the computation of the $\mathrm{Cl}$ for method with vertical diffusion cell. As the $90 \% \mathrm{Cl}$ was $85.18 \%-102.66 \%$, it was concluded that vertical diffusion cell method is not sensitive to changes in API particle size from the tested formulations.

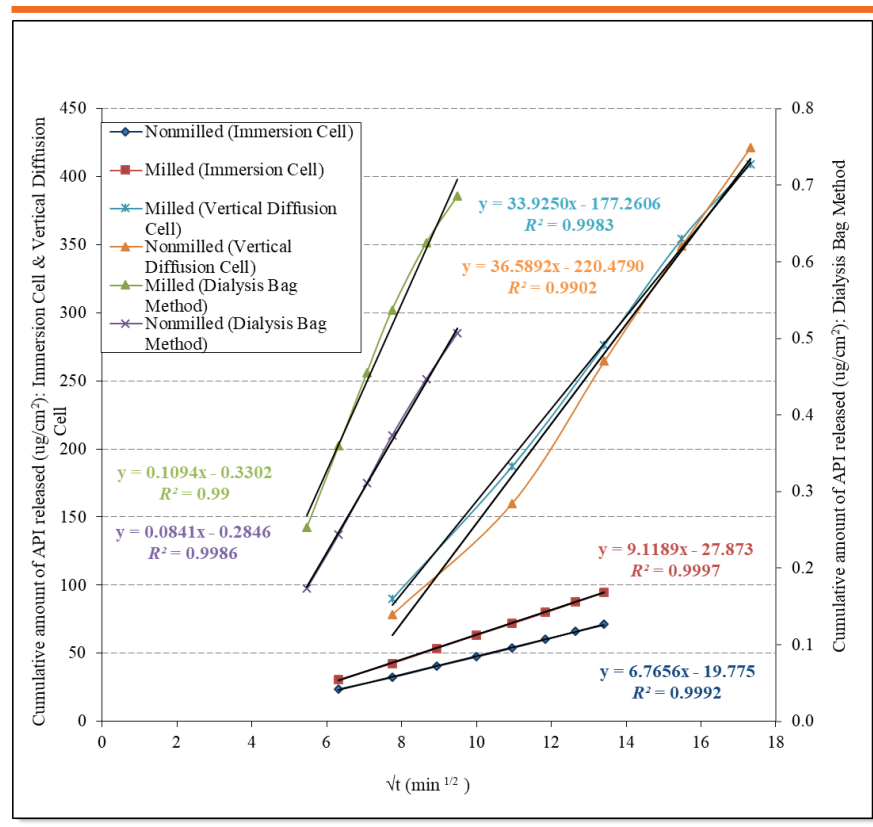

Figure 4. Drug release profiles from formulations with different particle size that were obtained using immersion cell, vertical diffusion cell, and dialysis bag method $(n=6)$. Black lines represent the slope. API, active pharmaceutical ingredient.

\section{The Repeatability of the Methods}

Repeatability of the methods was analyzed by comparing the average values of slopes (estimated in vitro release rates) for targeted $100 \%$ nanosuspension that were obtained bysensitivity testing on changes in concentration and particle size of the API. The percentage difference was

Table 2. Results of Selected IVRT Methods for Three Dexamethasone Nanosuspension Formulations with Different Dosage Strengths (50\%, 100\%, and 150\%)

\begin{tabular}{|c|c|c|c|c|c|}
\hline \multirow[b]{2}{*}{ IVRT Method } & \multicolumn{2}{|c|}{$90 \% \mathrm{Cl}$} & \multicolumn{2}{|c|}{ Average Slope } & \multirow[b]{2}{*}{ Difference $(\%)^{*}$} \\
\hline & $50 \%$ vs $100 \%$ & $100 \%$ vs $150 \%$ & $\begin{array}{c}\text { Concentration } \\
\text { Sensitivity }\end{array}$ & $\begin{array}{l}\text { Particle Size } \\
\text { Sensitivity }\end{array}$ & \\
\hline Immersion Cell Method & $68.60 \%-83.16 \%$ & $74.63 \%-93.22 \%$ & 8.6029 & 9.1189 & 5.82 \\
\hline Dialysis Bag Method & $49.43 \%-58.50 \%$ & $59.58 \%-89.89 \%$ & 0.0959 & 0.1094 & 13.15 \\
\hline Vertical Diffusion Cell Method & $41.49 \%-47.19 \%$ & $86.98 \%-103.84 \%$ & 35.489 & 33.925 & 4.51 \\
\hline
\end{tabular}

Cl, confidence interval; IVRT, in vitro release testing.

*Percent difference was calculated for the average slopes (estimated in vitro release rates) to determine repeatability of the methods. 
calculated for the average values of slopes (estimated in vitro release rates). The results, shown in Table 2 , indicate acceptable repeatability of all selected methods.

\section{CONCLUSION}

In the present study, three IVRT methods were developed for dexamethasone nanosuspensions. Immersion cell, dialysis bag methodology, and vertical diffusion cell methods were utilized to evaluate critical parameters of dexamethasone release from nanosuspension.

Diffusion of the drug across the PES membrane and CE dialysis membrane demonstrated low drug adsorption and low diffusional resistance. After an initial lag phase, the amount of drug released became proportional to the square root of time. The slope in the linear portion of the release curve was used as a measure of release rate. Although the dialysis bag membrane method revealed sufficient sensitivity to the tested parameters, higher variability in the results was observed.

In the last decade, IVRT using vertical diffusion cells has been a promising tool to assess the release of API from topical products; however, in this study, this method did not show sufficient sensitivity for dexamethasone released from nanosuspension due to the small surface of the membrane across which diffusion of the therapeutic agent occurs.

The immersion cell method had the best discriminatory power and repeatability for IVRT of dexamethasone from nanosuspension. Therefore, this method is the most appropriate for evaluation of dexamethasone release from nanoparticle-based therapeutic systems.

\section{ACKNOWLEDGMENTS}

We thank Jelena Tkalec who prepared the nanosuspension formulations.

\section{CONFLICT OF INTEREST}

The authors disclosed no conflicts of interest related to this article.

\section{REFERENCES}

1. Gupta, P.; Garg, S. Semisolid dosage forms for dermatological application. Pharm. Tech. 2002, 26, 144.

2. Jayaprakash, R.; Krishnakumar, K.; Dineshkumar, B.; Jose, R.; Nair, S. K. Nanosuspension in drug delivery-A review. Sch. Acad. J. Pharm. 2016, 5, 138-141.

3. Anusha, D.; Rao V. U. M.; Shekhar S. R. Review on nanosuspension technology. Int. J. Pharm. Dev. Tech. 2015, 5, 137-140.

4. Jug, M.; Hafner, A.; Lovrić J.; Lusina Kregar, M.; Pepić, I.; Vanić, Ž.; Cetina-Čižmek, B.; Filipović-Grčić, J. An overview of in vitro dissolution/release methods for novel mucosal drug delivery systems. J. Pharm. and Biomed. Anal. 2018, 147, 350-366. DOI: 10.1016/j.jpba.2017.06.072.

5. Klein, R. R.; Tao, J. Q.; Wilder, S.; Burchett, K.; Bui, Q.; Thakker, K. D. Development of an in vitro release test (IVRT) for a vaginal microbicide gel. Dissolution Technol. 2010, 17, 6-10. DOI: 10.14227/DT170410P6.

6. <1724> Semisolid drug products-performance tests. In The United States Pharmacopeia and National Formulary USP 37-NF 32; The United States Pharmacopeial Convention, Inc.: Rockville, MD, 2014; pp 1273-1284.

7. SUPAC-SS: Nonsterile Semisolid Dosage Forms. Scale-Up And Postapproval Changes: Chemistry, Manufacturing, and Controls; In Vitro Release Testing and In Vivo Bioequivalence Documentation; Guideance for Industry; U.S. Department of Health and Human Services, Food and Drug Administration, Center for Drug Evaluation and Research (CDER), U.S. 